\title{
A Dynamic Mechanistic Model of Perceptual Binding
}

\author{
Pavel Kraikivski ${ }^{1, *}$
}

1 Academy of Integrated Science, Division of Systems Biology, Virginia Polytechnic Institute and State University, Blacksburg, VA 24061 USA; pavelkr@vt.edu

* Correspondence: pavelkr@vt.edu

\begin{abstract}
The brain's ability to create a unified conscious representation of an object by integrating information from multiple perception pathways is called perceptual binding. Binding is crucial for normal cognitive function. Some perceptual binding errors and disorders have been linked to certain neurological conditions, brain lesions, and conditions that give rise to illusory conjunctions. However, the mechanism of perceptual binding remains elusive. Here, we present a computational model of binding using two sets of coupled oscillatory processes that are assumed to occur in response to two different percepts. We use the model to study the dynamic behavior of coupled processes to characterize how these processes can modulate each other and reach a temporal synchrony. We identify different oscillatory dynamic regimes that depend on coupling mechanisms and parameter values. The model can also discriminate different combinations of initial inputs that are set by initial states of coupled processes. Decoding brain signals that are formed through perceptual binding is a challenging task, but our modeling results demonstrate how crosstalk between two systems of processes can possibly modulate their outputs. Therefore, our mechanistic model can help one gain a better understanding of how crosstalk between perception pathways can affect the dynamic behavior of the systems that involve perceptual binding.
\end{abstract}

Keywords: Binding Problem; Perceptual Binding; Consciousness; Perception;

\section{Introduction}

Perceptual binding provides a unified conscious representation of an object that is described by several different perceptual features such as the object's shape, color, and location [1, 2]. Importantly, accumulated empirical evidence suggests that binding is critical for normal cognitive operation. Binding disorder occurs in damaged brains when patients cannot perceive more than one object at a time, have dissociations between different perception pathways, and have problems solving a discrimination task according to different percepts [3,4]. Also, illusory conjunctions are often referred to as examples of binding errors $[5,6]$. Thus, a normal cognitive operation requires appropriate integration of neural signals from different perception pathways.

Building binding models could help us better understand how our brain integrates information from different perception pathways to provide us with a unified and coherent conscious experience. Several models have been proposed to explain the mechanism of perceptual binding, among which the most frequently discussed is based on the neuronal synchrony or temporal correlation hypothesis [2, 7-9]. In Operational Architectonics, an operational synchrony among neuronal processes initiated in different brain regions is postulated to play a central role in binding spatially dispersed phenomenal features into a unified phenomenal object [10-12]. A temporal alignment that permits binding between a stimulus and ongoing spontaneous neural activity is a core assumption of Temporo-spatial Theory of Consciousness [13, 14]. Furthermore, an interdependence between information integration and consciousness has been postulated in several theories of consciousness [15-18]. For example, the Integrated Information Theory identifies consciousness as the ability of the neuronal system to integrate information to the level at which information is consciously accessible [19-21]. In addition, some attempts to give a computational explanation of binding have been made within the framework of classical neural networks [2]. However, much remains to be understood about the neuronal processes involved in perceptual binding. Moreover, many works have been devoted to provide a critical evaluation of the temporal synchrony hypothesis as well as arguments against the existence of the binding problem in principle [22-24].

In this work, we present a mathematical model of binding, which is based on our previous model of oscillatory processes, that exhibits the dynamic behavior isomorphic to a specific percept [25-27]. To study 
the binding mechanism, we used two sets of oscillatory processes that are bound via negative feedback loops. Two different binding interaction wiring schemes were analyzed. We studied how the sets of oscillatory processes modulated each other and found different regimes of modulated oscillations, which are represented in a two-parameter bifurcation diagram. Furthermore, we investigated how the system in these different regimes was capable of distinguishing different combinations of initial inputs (stimuli). We intend our dynamic model to help us understand how a possible mechanism of perceptual binding can be deduced from observable oscillating signals.

\section{Model and Methods}

Perceptual experiences of two individuals can be synchronized by the same stimulus; therefore, in principle, temporally correlated neuronal signals can be recorded in two non-interacting brains. In that case, however, the temporal synchronization is not sufficient to provide a unified conscious representation because the stimulus is still independently processed by each individual. Therefore, binding can occur only if there is a crosstalk between perception pathways that can interact and exchange information. Binding is thus detectable because the crosstalk between pathways may result in modulation and superposition of signals that can be analyzed. Here, we assume that direct interaction among perception pathways or corresponding processes is a necessary condition for binding, which can, in turn, induce temporal correlation, synchronization, or modulation among oscillating processes.

We use our previous framework in which a set of oscillating processes is used to represent a percept [25] such as a space or a position in the space. The spatial position is encoded in the relationships among processes denoted as $\vec{P}$, which is closely analogous to an intrinsic space as defined in the temporo-spatial theory of consciousness $[13,14]$. We also consider an attribute associated with the position in space such as brightness of a source at that position, which is assumed to be similarly encoded in the relationships among processes denoted as $\vec{Q}$. Thus, to study binding, we use two closed sets of processes: $\vec{P}=$ $\left(p_{1}, p_{2}, x_{1}, x_{2}\right)$ and $\vec{Q}=\left(q_{1}, q_{2}, y_{1}, y_{2}\right)$, which are described by the following system of equations:

$$
\begin{gathered}
\frac{d p_{1}}{d t}=\varepsilon p_{2}-p_{1}-x_{1}+f_{1}\left(q_{1}, q_{2}\right) \\
\frac{d p_{2}}{d t}=\varepsilon p_{1}-p_{2}-x_{2}+f_{2}\left(q_{1}, q_{2}\right) \\
\frac{d x_{1}}{d t}=p_{1}, \quad \frac{d x_{2}}{d t}=p_{2} \\
\frac{d q_{1}}{d t}=\alpha q_{2}-q_{1}-y_{1}+g_{1}\left(p_{1}, p_{2}\right) \\
\frac{d q_{2}}{d t}=\alpha q_{1}-q_{2}-y_{2}+g_{2}\left(p_{1}, p_{2}\right) \\
\frac{d y_{1}}{d t}=q_{1}, \quad \frac{d y_{2}}{d t}=q_{2}
\end{gathered}
$$

Where $\varepsilon$ and $\alpha$ are parameters describing the mutual interactions between $p$-processes and between $q$-processes correspondingly, see Figure $1 \mathrm{a}, \mathrm{b}$. The $f_{1}\left(q_{1}, q_{2}\right), f_{1}\left(q_{1}, q_{2}\right)$ and $g_{1}\left(p_{1}, p_{2}\right), g_{1}\left(p_{1}, p_{2}\right)$ functions describe the binding between the $\vec{P}$ and $\vec{Q}$ sets of processes. Generally, a function that depends on a difference between oscillating variables can be used to achieve a synchronization of two oscillators [28] (pp. 123-136). Two oscillators that communicate the phase to one another can be drawn into synchrony over time. In System (1), we assume that the interaction among processes is realized via negative feedback loops (see Figures $1 \mathrm{a}, \mathrm{b}$ that show two possible coupling mechanisms between the $\vec{P}$ and $\vec{Q}$ sets of processes). Mathematically, we consider the following two interaction schemes: (a) $f_{1}\left(q_{1}, q_{2}\right)=q_{1}$, 
$f_{2}\left(q_{1}, q_{2}\right)=q_{2}, g_{1}\left(p_{1}, p_{2}\right)=-p_{1}, g_{2}\left(p_{1}, p_{2}\right)=-p_{2}$, where this formulation corresponds to the mechanism shown in Figure 1a and (b) $f_{1}\left(q_{1}, q_{2}\right)=q_{1}-q_{2}, f_{2}\left(q_{1}, q_{2}\right)=q_{2}-q_{1}, g_{1}\left(p_{1}, p_{2}\right)=p_{2}-p_{1}, g_{2}\left(p_{1}, p_{2}\right)=$ $p_{1}-p_{2}$ is according to the mechanism shown in Figure $1 \mathrm{~b}$.

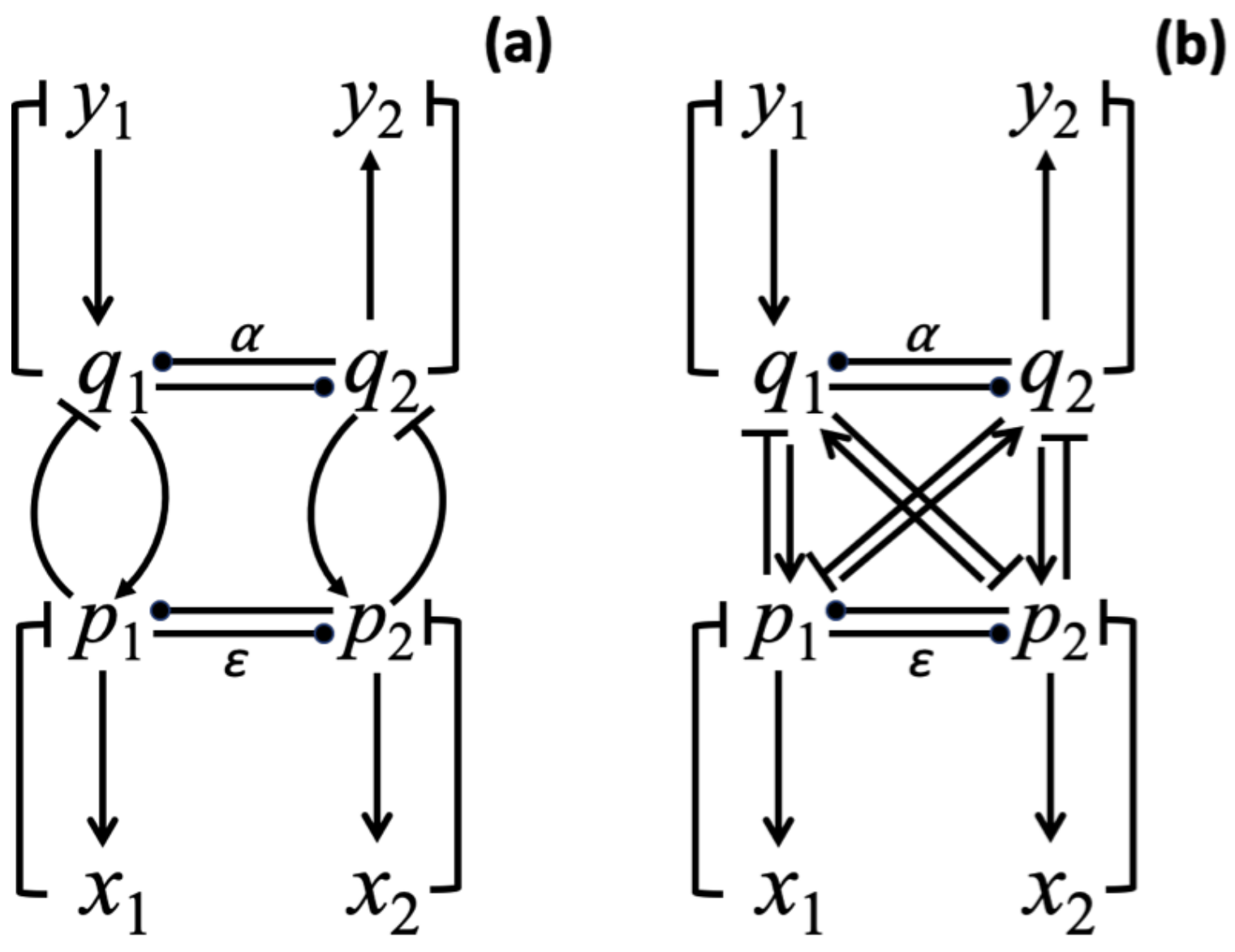

Figure 1. Two different influence schemes for processes described by the system equations (1). (a) Binding between $\vec{P}$ and $\vec{Q}$ sets of processes is gained through interaction of $p_{1}$ with $q_{1}$ and $p_{2}$ with $q_{2}$ processes. In neural systems such specific winning links can be established via training in response to different combinations of input stimuli. Here, negative feedback loops are used to realize connections between $p_{1}$ and $q_{1}$ as well as between $p_{2}$ and $q_{2}$. (b) Binding between $\vec{P}$ and $\vec{Q}$ sets of processes in which all $p_{1}, p_{2}, q_{1}, q_{2}$ processes are mutually interconnected via negative feedback loops. Arrow-headed lines represent a positive influence and bar-headed lines represent a negative influence. The dot-headed lines represent positive or negative influence depending on the sign of the $\alpha$ and $\varepsilon$ parameters.

We assume that the $\vec{P}$ and $\vec{Q}$ processes occur in response to two different percepts. For example, the state of $\left(p_{1}, p_{2}\right)$ can represent the processes in response to the specific position selected by attention, which is indicated by a blue circle in Figure 2, and the state of $\left(q_{1}, q_{2}\right)$ can represent the processes in response to the presence or absence of a light stimulus at the selected location (shown by a star sign or black dot in Figure 2). Hence, we distinguish the position of a spot that can be either dark or bright by focusing our attention either on the bright spot (e.g., case (i) in Figure 2) or on the dark spot (e.g., case (ii) in Figure 2). Therefore, our model represents perceptual binding occurring for two percepts that include the position in space and an attribute that is assigned to each position. The position selection is represented by the $p_{1}$ and $p_{2}$ processes. The initial value for the $p_{1}$ or $p_{2}$ variable is set to 1 if the corresponding position is selected or to zero otherwise (see Figure 2). Similarly, the initial value for the process $q_{1}$ or $q_{2}$ is set to 1 if a light stimulus is present, otherwise the initial value for $q_{1}$ or $q_{2}$ is set to zero. All 
corresponding initial values for $p_{1}, p_{2}, q_{1}, q_{2}$ processes for cases (i)-(viii) are shown in Figure 2. Simulation results of these cases are provided in the Results section of this manuscript.

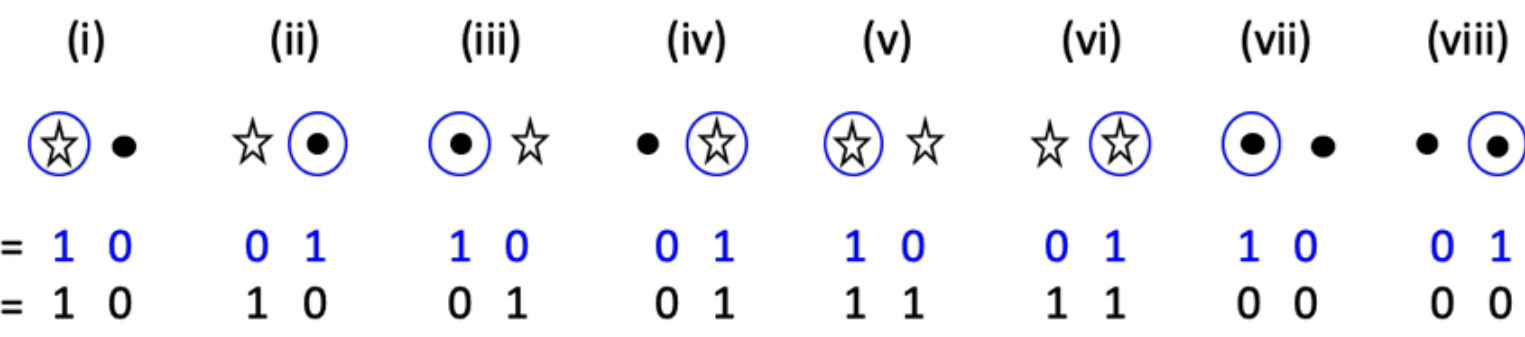

Figure 2. Seven different combinations of two distinct types of inputs representing percepts and the corresponding initial values of $\left(p_{1}, p_{2}\right)$ and $\left(q_{1}, q_{2}\right)$ processes. Each combination consists of two possible positions and the presence/absence of a light stimulus at these positions. The presence of a light stimulus at a position is indicated by the star sign and the absence of light is indicated by the black dot. A specific position is assumed to be selected by attention, which is indicated by the blue circle. For example, for case (i), the focus is on the bright spot, while for case (ii), the focus is on the dark spot.

System (1) consists of a system of linear differential equations that has a solution that can be, in principle, expressed in algebraic form. However, eight eigenvalues and eigenvectors cannot be written in a concise form to fit into this text and be easily analyzed. Therefore, here, we present numerical solutions obtained for different initial conditions and parameter values that produce distinct numerical results. XPP/XPPAUT software (http://www.math.pitt.edu/ bard/xpp/xpp.html, accessed on 12th January 2022) was used to solve System (1) and compute two-parameter bifurcation diagrams. XPP codes that were used to produce all results presented in this work are provided in Appendix A.

\section{Results}

First, we analyzed System (1) by considering the interaction scheme shown in Figure 1a. We explored solutions of System (1) for different values of $\varepsilon$ and $\alpha$ parameters to identify distinct dynamic regimes that the system of coupled processes can exhibit. Thus, $\varepsilon$ and $\alpha$ serve as bifurcation parameters of the system. As shown in Figure 3, the dynamic behavior of the $\vec{P}$ and $\vec{Q}$ processes depended on $\varepsilon$ and $\alpha$ parameter values. The feedback loops connecting the two sets of processes induced a complex mutual modulation among the interacting processes. Variations in amplitude, frequency, and temporal relationships among processes depending on parameter values were observed. Amplitude modulation or distortion was also observed for two coupled oscillators when the coupling was not strong enough to bring the phases of two oscillators into synchrony over time [28] (pp. 123-136). Similarly, the strong amplitude modulation in System (1) occurred when one parameter, either $\varepsilon$ or $\alpha$, was much smaller than the other (see Figure 3e, f). Therefore, amplitude distortion is likely to be observed in a system that is composed of two interacting subsystems such that one subsystem is described by weak internal coupling parameters and the other is described by strong internal coupling parameters. Interestingly, we also observed small amplitude variations when both $\varepsilon$ and $\alpha$ had values close to -1 . These variations also occurred due to interactions between the $\vec{P}$ and $\vec{Q}$ processes. When the $\vec{P}$ and $\vec{Q}$ processes are decoupled, the amplitude variations disappear. Thus, the amplitude distortion in recoded signals could indicate binding. 

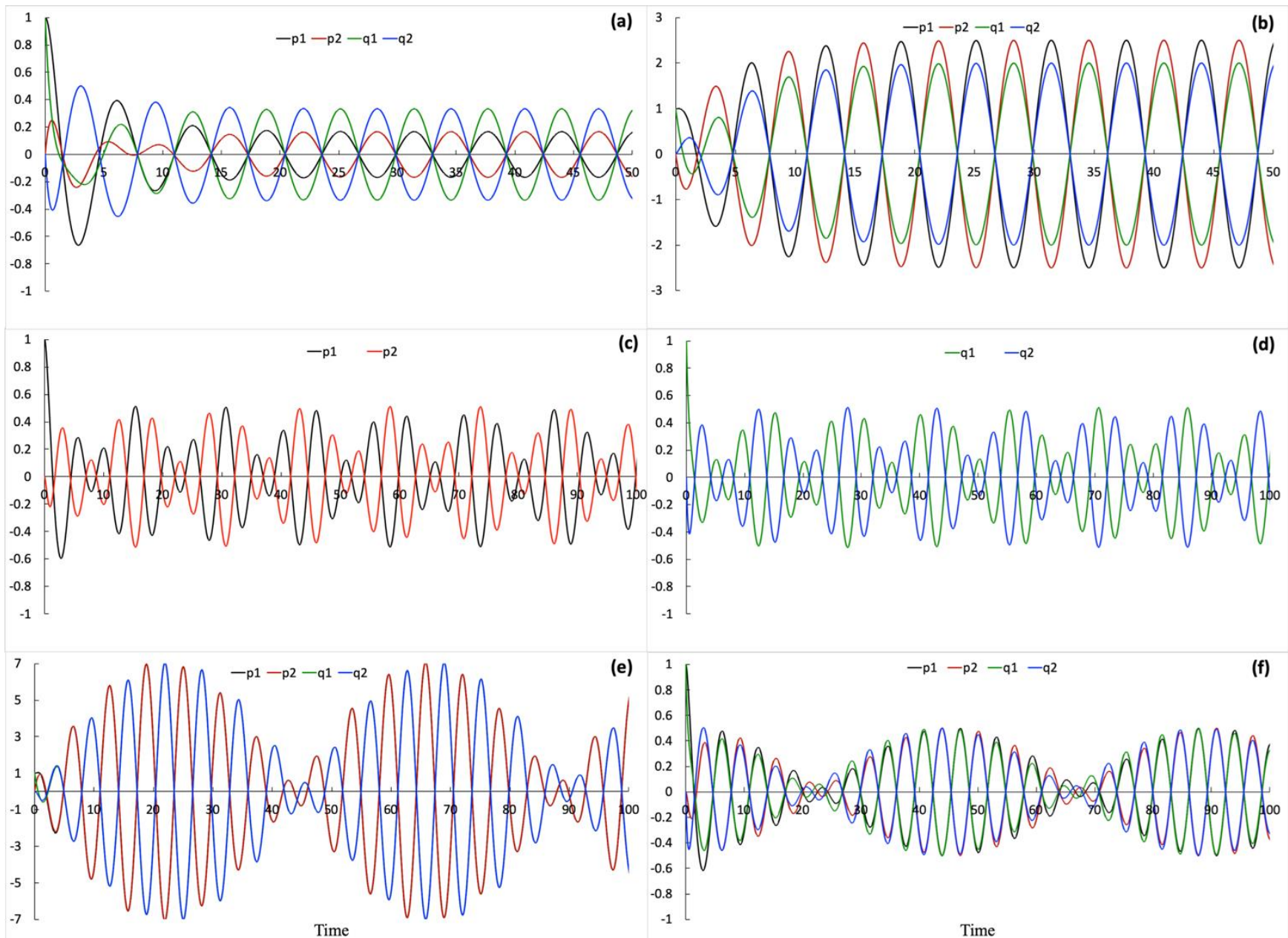

Figure 3. Oscillatory behavior observed in the dynamic system of coupled processes. Oscillations of $\left(p_{1}, p_{2}\right)$ and $\left(q_{1}\right.$, $q_{2}$ ) processes are obtained using the following parameter values: (a) $\varepsilon=1, \alpha=-1.5 ; \quad$ (b) $\varepsilon=-1.8, \alpha=0.25$; (c) and (d) $\varepsilon=-0.1, \alpha=-1.9$, where the $\left(p_{1}, p_{2}\right)$ and $\left(q_{1}, q_{2}\right)$ processes are shown on separate figure panels for better visualization; (e) $\varepsilon=1.99, \alpha=0.01$, note that shortly after the starting values the $\left(p_{1}, p_{2}\right)$ and $\left(q_{1}, q_{2}\right)$ processes begin and continue to overlap over time; $(\mathbf{f}) \varepsilon=-0.01, \alpha=-1.99$. All simulations are obtained using the same initial conditions: $\left(p_{1}, p_{2}\right.$, $\left.q_{1}, q_{2}\right)=(1,0,1,0)$ that corresponds to case (i) in Figure 2 , and $\left(x_{1}, x_{2}, y_{1}, y_{2}\right)=(0,0,0,0)$.

Here, we only present periodic solutions with sustained oscillations; however, our system also exhibits damped oscillations and unstable sources or oscillations with growing amplitude as well. The periodic solutions of System (1) with sustained oscillations were found for the following ranges of parameters:

$$
\begin{gathered}
\alpha=-\frac{\varepsilon+2}{\varepsilon+1} \text { for }-2<\varepsilon \leq-\sqrt{2} \text { and } 0<\varepsilon \leq \sqrt{2} \\
\alpha=\frac{\varepsilon-2}{\varepsilon-1} \text { for }-\sqrt{2} \leq \varepsilon<0 \text { and } \sqrt{2} \leq \varepsilon<2 \\
\alpha=-\varepsilon-2 \text { for }-2<\varepsilon<0 \\
\alpha=-\varepsilon+2 \text { for } 0<\varepsilon<2 .
\end{gathered}
$$

The corresponding two-parameter bifurcation diagram is shown in Figure 4. The diagram is obtained numerically as explained in the Methods section and agrees with the analytical solutions described by the System of Equations (2). The parameter ranges marked as (i) and (iii) in Figure 4 correspond to the first 
equation in System (2); the regions marked as (iv) and (vi) in Figure 4 correspond to the second equation in System (2); the line marked as (ii) in Figure 4 corresponds to the third equation in System (2) and the line labeled with (v) in Figure 4 corresponds to the last equation in System (2). Oscillations were observed with varying amplitudes for parameter values along the (ii) and (v) lines shown in Figure 4. Figures 3c, $\mathrm{d}$, and $\mathrm{f}$ provide examples of oscillations obtained using parameter values from region (ii) and Figure $3 \mathrm{e}$ shows simulations obtained using parameter values from region (v). Oscillations produced using parameter values from regions (i) and (iii) are shown in Figure 3a and Figure 3b, respectively. Oscillations for parameter values taken in regions (iv) and (vi) have constant amplitude similar to those shown in Figures $3 \mathrm{a}, \mathrm{b}$ but $p_{1}$ and $q_{1}$ oscillate in phase with $p_{2}$ and $q_{2}$, respectively (not shown). Overall, System (1) combined with the interaction scheme shown in Figure 1a produces a diverse repertoire of periodic solutions with sustained oscillations that depend on the system's parameters.

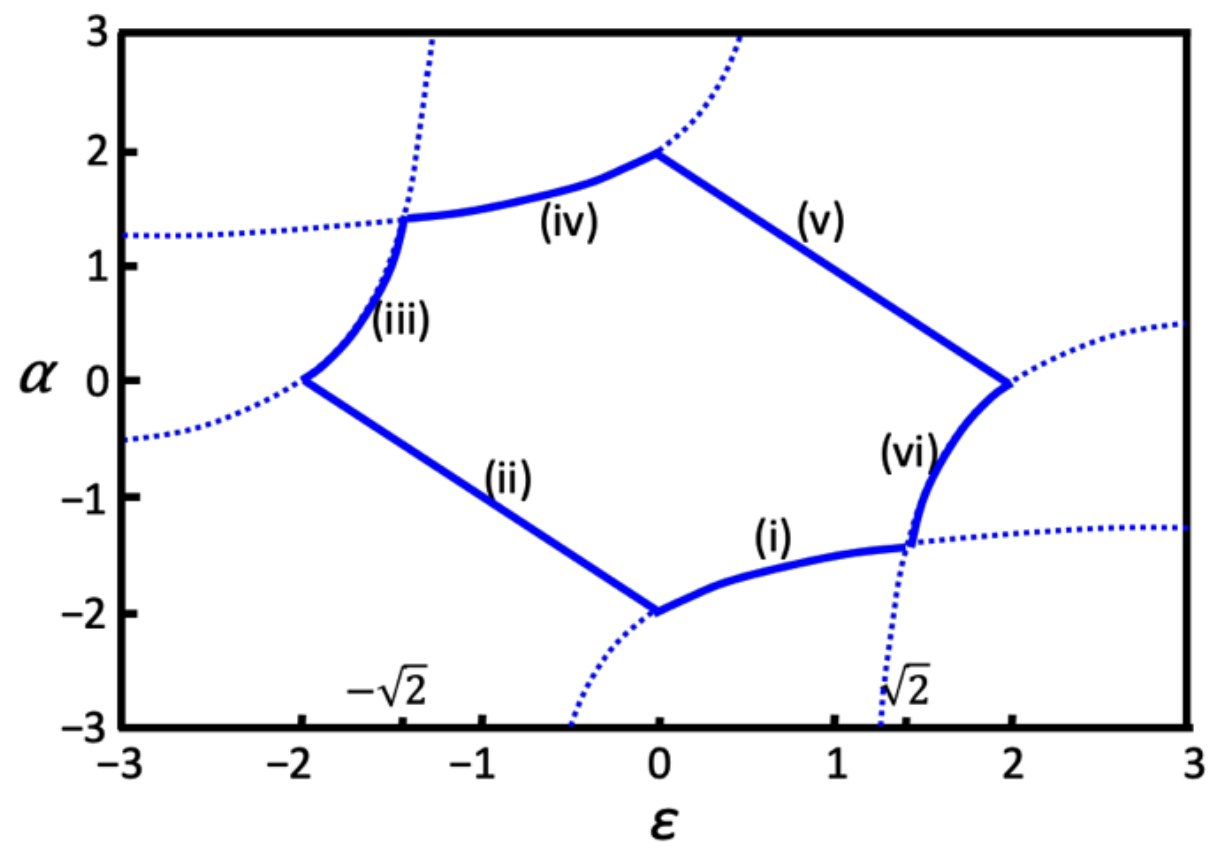

Figure 4. Two-parameter bifurcation diagram for the interaction scheme shown in Figure 1a. Oscillations occur for $\varepsilon$ and $\alpha$ parameter values along solid curves marked as (i)-(v). To demonstrate agreement between numerical and analytical solutions, the dotted curves are drawn by plotting the following functions: $\alpha=-(\varepsilon+2) /(\varepsilon+1)$ and $\alpha=(\varepsilon-2) /(\varepsilon$ $-1)$, which overlap with solid curves (iii), (i) and (iv), (vi) obtained numerically in corresponding regions.

Next, we fixed the $\varepsilon$ and $\alpha$ parameter values and investigated the solutions of System (1) depending on the different initial conditions that are shown in Figure 2. $\varepsilon$ and $\alpha$ parameter values were taken from region (ii), shown in Figure 4, which are also described by the third equation in System (2). For these parameter values, the system of processes exhibits sustained oscillations for both interaction schemes shown in Figures 1a and b. Thus, we can compare how different interaction schemes perform in solving a discrimination task by differentiating inputs shown in Figure 2.

Six conditions (i)-(vi) shown in Figure 2 produce distinct dynamic relationships among $p_{1}, p_{2}, q_{1}$, $q_{2}$ processes (see Figure 5). However, two conditions (vii) and (viii), shown in Figure 2, produce the same dynamic relationships among processes as obtained for the (v) and (vi) conditions, respectively. Therefore, the system can discriminate (i)-(vi) initial inputs but cannot discriminate (vii) and (viii) from (v) and (vi) inputs. The latter means that the position in space that is homogeneously bright is identical to the same position in space that is homogeneously dark. Perhaps, these inputs can be discriminated if more complex interactions representing binding or a system with more states and hierarchical levels of binding interactions between these states are used. 

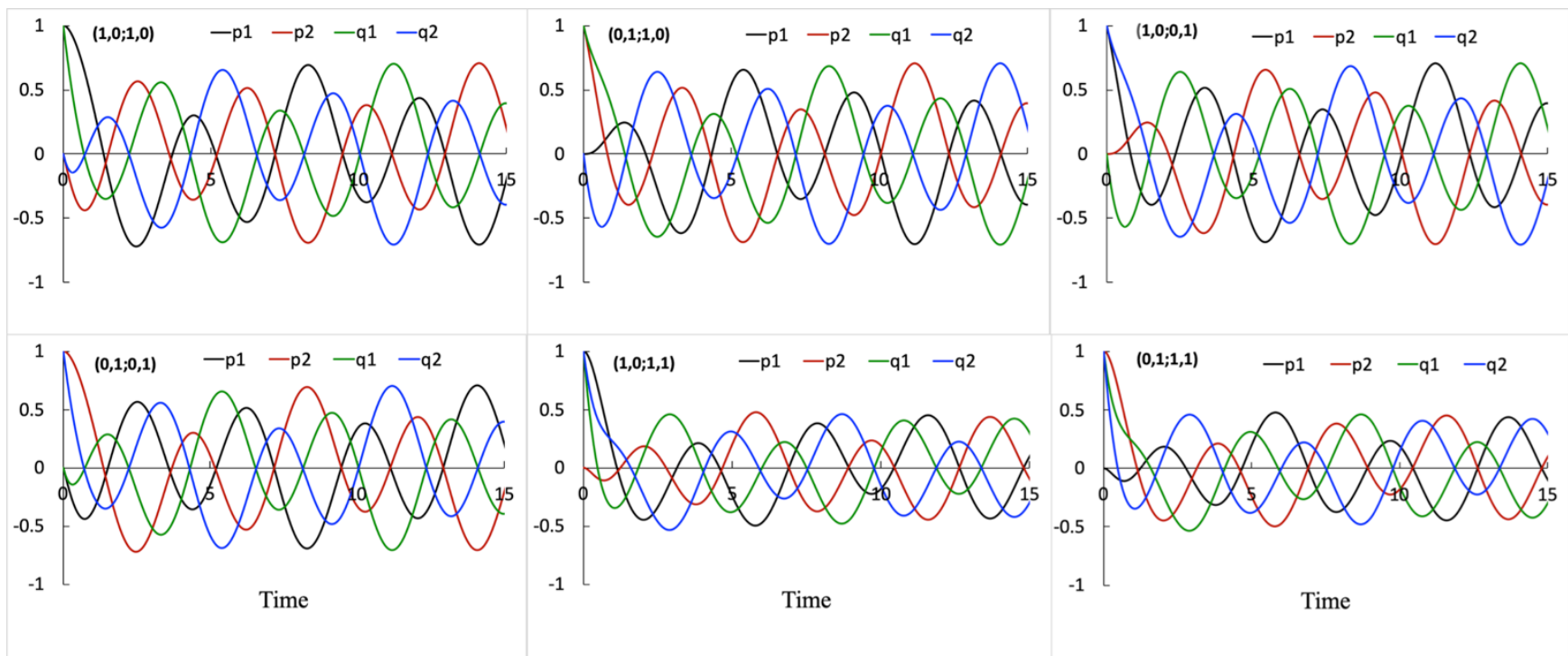

Figure 5. The change in dynamic relationships among $p_{1}, p_{2}, q_{1}, q_{2}$ processes depend on initial conditions. Six initial conditions (i)-(vi) shown in Figure 2 are used to produce these simulation results. These conditions induce distinct dynamic relationships among processes and, thereby, can be discriminated by the system. The initial conditions are indicated in the figures as two pairs of digits corresponding to initial values of $\left(p_{1}, p_{2} ; q_{1}, q_{2}\right)$ shown in parenthesis at the upper left corner of each figure panel. All simulations are obtained using the interaction scheme shown in Figure 1a and the following parameter values: $\varepsilon=-1, \alpha=-1$.

Solving and analyzing System (1) for the second interaction scheme shown in Figure 1b, we also identified $\varepsilon$ and $\alpha$ parameter values for which the periodic solutions with sustained oscillations are obtained. A two-parameter bifurcation diagram that summarizes parameter ranges with periodic solutions is shown in Figure 6. Oscillations for parameter values along the solid line in Figure 6 are qualitatively similar to those that are shown in Figure 5 and obtained for the same range of parameters: $\alpha=-\varepsilon-2$ for $-2<\varepsilon<0$, however, the frequency of oscillations is higher (see Figure S1 in Supplementary Materials). Also, System (1) combined with the interaction scheme in Figure $1 \mathrm{~b}$ performs equally well to that of the scheme shown in Figure 1a on the task to discriminate different inputs that are shown in Figure 2. However, comparing two-parameter bifurcation diagrams in Figures 5 and 6, the diagram in Figure 6 shows significantly more parameter ranges where the system exhibits sustained oscillations. Therefore, despite the fact that the interaction scheme in Figure $1 \mathrm{~b}$ has more interactions than the interaction scheme shown in Figure 1a, the latter, simpler interaction scheme produces a more diverse dynamic behavior of the system. Thus, the more comprehensive interaction network that integrates information from many network nodes does not necessarily result in a more diverse dynamic repertoire. 


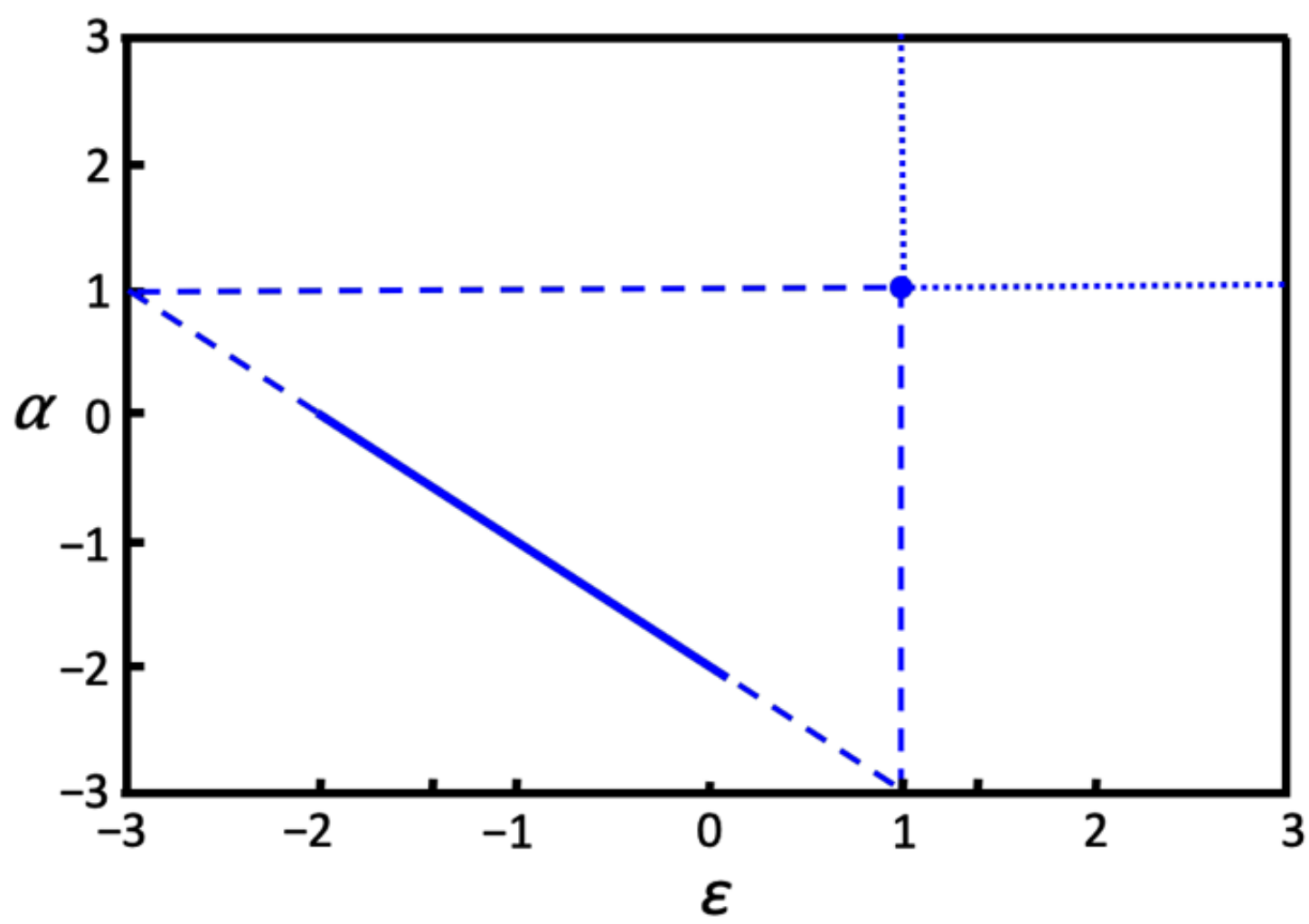

Figure 6. Two-parameter bifurcation diagram for the interaction scheme shown in Figure 1b. The solid line, $\alpha=-\varepsilon-$ 2 for $-2<\varepsilon<0$, shows the parameter values where both $\vec{P}=\left(p_{1}, p_{2}, x_{1}, x_{2}\right)$ and $\vec{Q}=\left(q_{1}, q_{2}, y_{1}, y_{2}\right)$ oscillate and the relationships between $p_{1}$ and $p_{2}$ and $q_{1}$ and $q_{2}$ are maintained over time. For these parameter values, $\vec{P}$ and $\vec{Q}$ oscillate out of phase and with varying amplitudes. Dashed lines show the parameter values where oscillations exist but either the relationships between p-processes or q-processes are no longer maintained (diagonal dashed lines), or only one set of processes oscillate while another set of processes does not exhibit oscillatory dynamics (vertical and horizontal dashed lines). Dotted lines show parameter ranges where oscillations exist only if either $\vec{P}$ or $\vec{Q}$ starts with zero initial conditions and, thus, only p-processes or q-processes oscillate, which is equivalent to the situation when $\vec{P}$ and $\vec{Q}$ are decoupled and independent. At the point $(\alpha=1, \varepsilon=1)$, both p-processes and q-processes oscillate in phase with constant amplitudes.

\section{Discussion}

Mechanistic modeling has become a very popular tool that allows one to not simply describe the system components but to also analyze, understand, and explain the dynamic behavior of the system. This mechanistic approach has been successfully applied to modelling nerve action potential [29], neurodynamics in the olfactory system [30], dynamics of ecological networks [31], molecular signaling pathways [32], and complex molecular mechanisms determining cell fate [28, 33-36]. However, building mechanistic models of consciousness is undoubtedly one of the most challenging tasks. By using our previous dynamic modeling framework to describe a percept [25-27], here, we developed a dynamic mechanistic model of perceptual binding.

The perceptual binding concept is compatible with the Integrated Information and Temporo-Spatial theories of consciousness as well as with some classical neural network models [2, 9, 13-16]. However, as put forward by von der Malsburg, for example, the classical neural network models interpret a brain state as a static vector ignoring the fact that recorded neural signals are not constant over any fixed time scale [2]. By contrast, in our model, the states are encoded in the dynamical processes that continuously 
alternate, yet their specific relationships that encode information are maintained over time. This continuous realization of specific relationships among processes in the system is an important concept in our modeling framework. The main assumption of our approach is that consciousness is a dynamical process, not a capacity, memory, or information, as elaborated in Ref. [37].

Our dynamic approach is comparable to Freeman's framework developed to describe population neurodynamics in the olfactory system [30]. His system of ordinary differential equations constructed in conformance with the anatomical and physiological properties of the olfactory system has been successful in explaining electrophysiological recordings of impulse responses. Different oscillations have been observed including complex and highly dimensional oscillations with varying amplitudes and a pattern that repeats itself (see Figure 6 on page 301 in Ref. [30]). While our simulation results may not be directly comparable with the dynamic behavior of neuronal systems or with electroencephalographic brain recordings, our model provides a qualitative representation of how binding can influence and change neural oscillations. Although, we analyzed a simple system in which the space is represented by two points described by the $\left(p_{1}, p_{2}\right)$ processes and each point was characterized only by two states $\left(q_{1}, q_{2}\right)$, the system can be scaled to $n$-points each with $\mathrm{m}$-states $(\mathrm{n}, \mathrm{m}>2)$ as shown in Refs. [25, 26]. However, the application of sets with many states to investigate binding would only complicate the analysis and interpretation of results.

We analyzed a system of two sets of processes representing two different percepts and found that the system exhibits different dynamic behavior depending on initial conditions (see Figure 5) and is capable of distinguishing different combinations of initial inputs shown in Figure 2. Therefore, our approach can be an alternative to classical neural networks that fail to solve a discrimination task in Frank Rosenblatt's example with four neurons where two neurons learn to recognize the object shape and the other two indicate the position of objects $[2,38]$. The output reads of such a four-neuron classical network include two shapes (e.g., square, triangle) and two positions (left, right), however, whether a specific shape is on the left side or on the right side remains indistinguishable.

We investigated two interaction schemes (see Figure 1a, b) describing binding between the sets of processes. For both wiring schemes, different dynamic oscillatory regimes were identified. Remarkably, despite the comprehensive level of interactions among processes and, therefore, a higher level of information integration, the interaction scheme shown in Figure $1 \mathrm{~b}$ does not result in overly complex dynamic behavior, as opposed to the interaction scheme in Figure 1a, which produces a more diverse repertoire of oscillating regimes (see Figures 3, 4, and 6). This result appears to be opposite to what would be expected in Integrated Information Theory, which identifies consciousness with the ability of the system to integrate information.

In conclusion, our mechanistic model can help one gain a better understanding of how binding can affect the dynamic behavior of the systems that involve perceptual binding. For example, our results suggest that amplitude modulation or distortion in recoded signals could be used to detect binding and reveal some properties of interacting subsystems. Furthermore, binding cannot be merely the result of synchronization of signals or temporal correlation that can spontaneously occur or be set in two non-interacting systems; binding may only occur when processes interact, resulting in modulation and superposition of signals. 


\section{Supplementary Materials:}
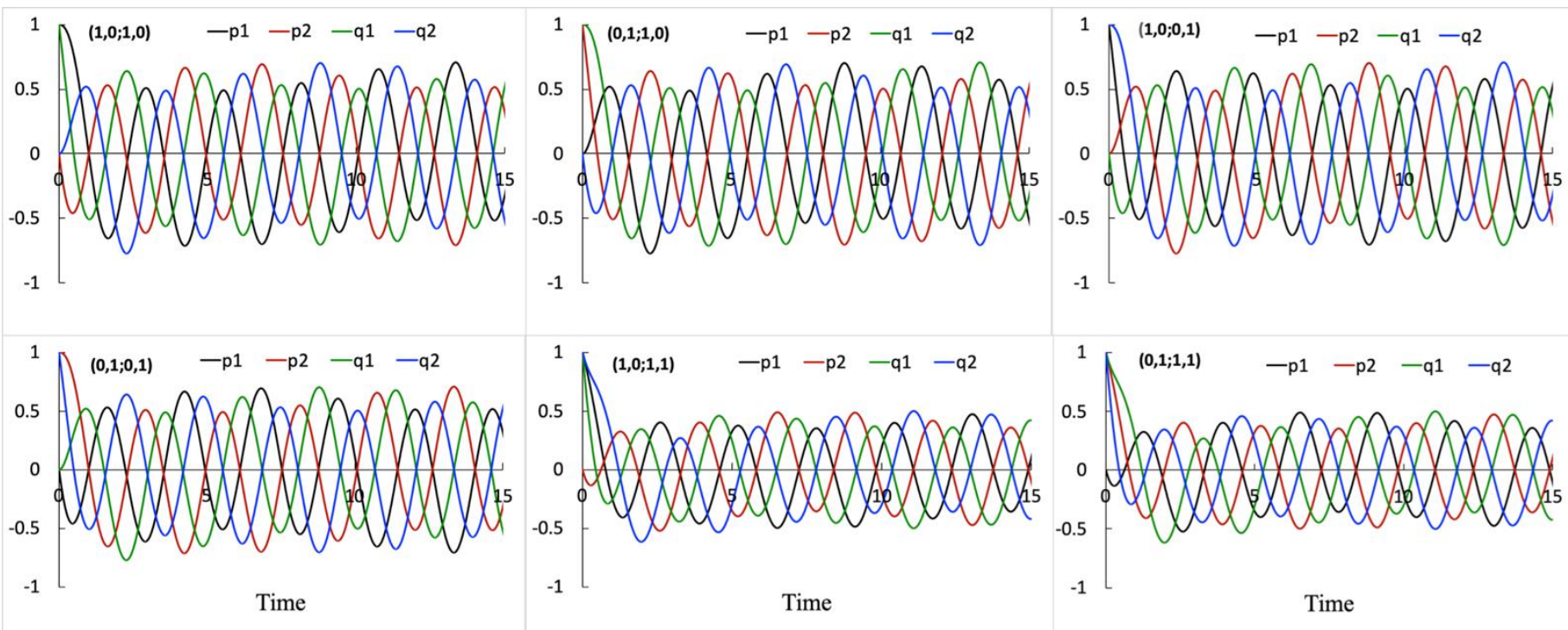

Figure S1: Dynamic relationships among processes depending on the initial conditions. Simulations are obtained using the interaction scheme shown in Figure $1 \mathrm{~b}$ and the following parameter values: $\varepsilon=-1, \alpha=-1$.

Conflicts of Interest: The author declares no conflict of interest. 


\section{Appendix A}

The XPPAUT code A was used to simulate results in Figures 3, 4, and 5.

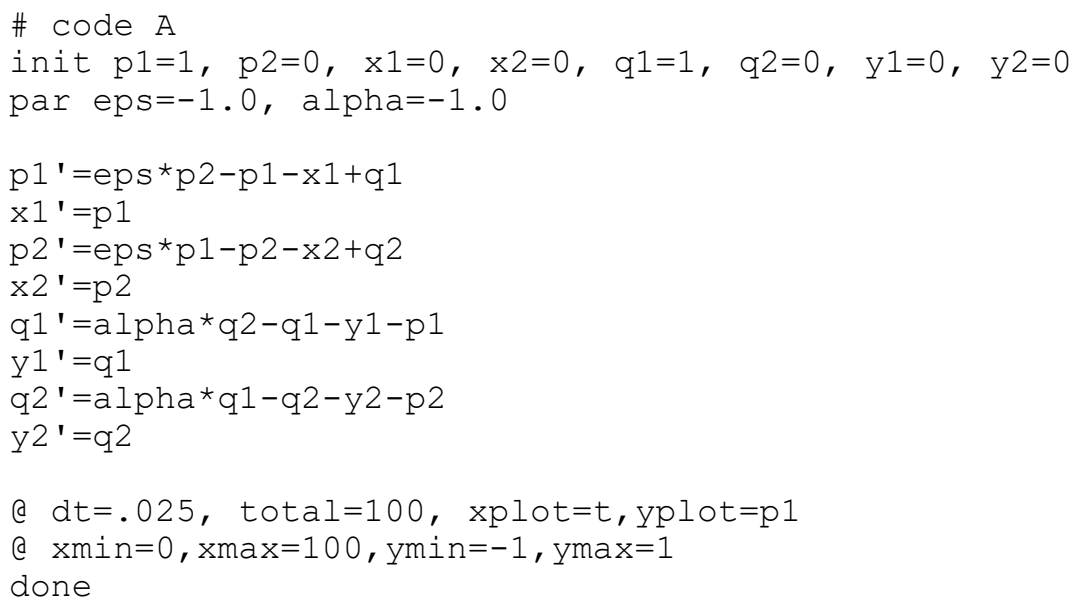

The XPPAUT code B was used to simulate results in Figure 6 and Figure S1 in the Supplementary Materials.

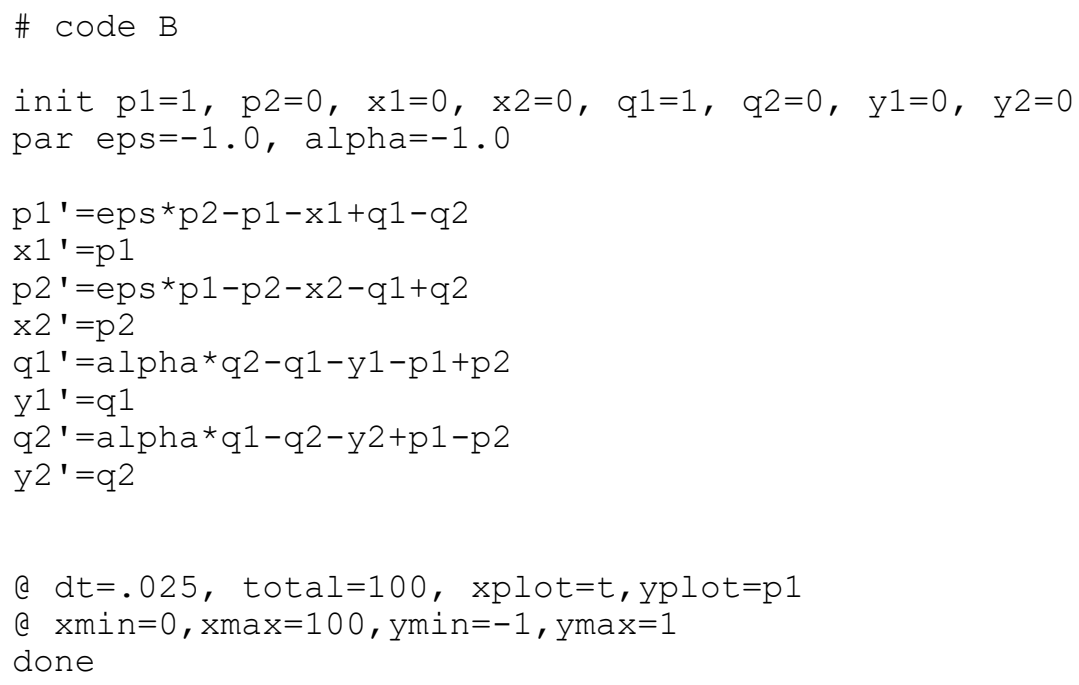

\section{References}

1. Roskies, A. L., The binding problem. Neuron 1999, 24, (1), 7-9, 111-25.

2. von der Malsburg, C., The what and why of binding: the modeler's perspective. Neuron 1999, 24, (1), 95-104, 11125.

3. Goodale, M. A.; Milner, A. D.; Jakobson, L. S.; Carey, D. P., A neurological dissociation between perceiving objects and grasping them. Nature 1991, 349, (6305), 154-6.

4. Goodale, M. A.; Meenan, J. P.; Bulthoff, H. H.; Nicolle, D. A.; Murphy, K. J.; Racicot, C. I., Separate neural pathways for the visual analysis of object shape in perception and prehension. Curr Biol 1994, 4, (7), 604-10.

5. Wolfe, J. M.; Cave, K. R., The psychophysical evidence for a binding problem in human vision. Neuron 1999, 24, (1), 11-7, 111-25. 
6. Treisman, A., Solutions to the binding problem: progress through controversy and convergence. Neuron 1999, 24, (1), 105-10, 111-25.

7. Gray, C. M., The temporal correlation hypothesis of visual feature integration: still alive and well. Neuron 1999, 24, (1), 31-47, 111-25.

8. Singer, W., Neuronal synchrony: a versatile code for the definition of relations? Neuron 1999, 24, (1), 49-65, 111-25.

9. von der Malsburg, C., Binding in models of perception and brain function. Curr Opin Neurobiol 1995, 5, (4), 520-6.

10. Fingelkurts, A. A.; Fingelkurts, A. A.; Neves, C. F. H., Phenomenological architecture of a mind and Operational Architectonics of the brain: the unified metastable continuum Journal of New Mathematics and Natural Computing 2009, 5, (1), 221-244.

11. Fingelkurts, A. A.; Fingelkurts, A. A., Mind as a nested operational architectonics of the brain. Physics of Life Reviews 2012, 9, (1), 49-50.

12. Fingelkurts, A. A.; Fingelkurts, A. A.; Neves, C. F. H., Consciousness as a phenomenon in the operational architectonics of brain organization: Criticality and self-organization considerations. Chaos, Solitons E Fractals 2013, $55,13-31$

13. Northoff, G., What the brain's intrinsic activity can tell us about consciousness? A tri-dimensional view. Neurosci Biobehav R 2013, 37, (4), 726-738.

14. Northoff, G.; Huang, Z. R., How do the brain's time and space mediate consciousness and its different dimensions? Temporo-spatial theory of consciousness (TTC). Neurosci Biobehav R 2017, 80, 630-645.

15. Seth, A. K.; Izhikevich, E.; Reeke, G. N.; Edelman, G. M., Theories and measures of consciousness: An extended framework. PNAS 2006, 103, (28), $10799-10804$.

16. Tononi, G.; Edelman, G. M., Consciousness and complexity. Science 1998, 282, (5395), 1846-51.

17. Dehaene, S.; Changeux, J. P., Experimental and theoretical approaches to conscious processing. Neuron 2011, 70, (2), 200-27.

18. Dehaene, S.; Charles, L.; King, J. R.; Marti, S., Toward a computational theory of conscious processing. Curr Opin Neurobiol 2014, 25, 76-84.

19. Tononi, G., An information integration theory of consciousness. BMC Neuroscience 2004, 5, 42.

20. Tononi, G., Consciousness as Integrated Information: a Provisional Manifesto. Biol Bull-Us 2008, 215 , (3), $216-242$.

21. Tononi, G., Information integration: its relevance to brain function and consciousness. Arch Ital Biol 2010, 148, (3), 299-322.

22. Shadlen, M. N.; Movshon, J. A., Synchrony unbound: a critical evaluation of the temporal binding hypothesis. Neuron 1999, 24, (1), 67-77, 111-25.

23. Riesenhuber, M.; Poggio, T., Are cortical models really bound by the "binding problem"? Neuron 1999, 24, (1), 87$93,111-25$.

24. Ghose, G. M.; Maunsell, J., Specialized representations in visual cortex: a role for binding? Neuron 1999, 24, (1), 79$85,111-25$.

25. Kraikivski, P., Systems of Oscillators Designed for a Specific Conscious Percept. New Mathematics and Natural Computation 2020, 16, 73-88.

26. Kraikivski, P., Implications of Noise on Neural Correlates of Consciousness: A Computational Analysis of Stochastic Systems of Mutually Connected Processes. Entropy 2021, 23, (5), 583.

27. Kraikivski, P., Building Systems Capable of Consciousness. Mind \& Matter 2017, 15, 185-195. 
28. Kraikivski, P., Case Studies in Systems Biology. 1 ed.; Springer, Cham: 2021; p 310.

29. Hodgkin, A. L.; Huxley, A. F., A quantitative description of membrane current and its application to conduction and excitation in nerve. J Physiol 1952, 117, (4), 500-44.

30. Freeman, W. J.; SpringerLink (Online service), Neurodynamics: An Exploration in Mesoscopic Brain Dynamics. In Perspectives in Neural Computing,, Springer,: London, 2000; pp X, 398p.

31. Loeuille, N.; Loreau, M., Evolutionary emergence of size-structured food webs. Proc Natl Acad Sci U S A 2005, 102, (16), 5761-6.

32. Jalihal, A. P.; Kraikivski, P.; Murali, T. M.; Tyson, J. J., Modeling and analysis of the macronutrient signaling network in budding yeast. Mol Biol Cell 2021, 32, (21), ar20.

33. Kraikivski, P.; Chen, K. C.; Laomettachit, T.; Murali, T. M.; Tyson, J. J., From START to FINISH: computational analysis of cell cycle control in budding yeast. NPJ Syst Biol Appl 2015, 1, 15016.

34. Tyson, J. J.; Laomettachit, T.; Kraikivski, P., Modeling the dynamic behavior of biochemical regulatory networks. J Theor Biol 2019, 462, 514-527.

35. Tyson, J. J.; Novak, B., A Dynamical Paradigm for Molecular Cell Biology. Trends Cell Biol 2020.

36. Jung, Y.; Kraikivski, P.; Shafiekhani, S.; Terhune, S. S.; Dash, R. K., Crosstalk between Plk1, p53, cell cycle, and G2/M DNA damage checkpoint regulation in cancer: computational modeling and analysis. NPJ Syst Biol Appl 2021, 7, (1), 46.

37. James, W., Does 'Consciousness' Exist? The Journal of Philosophy, Psychology and Scientific Methods 1904, 1, (18), 477491.

38. Rosenblatt, F., Principles of neurodynamics; perceptrons and the theory of brain mechanisms. Spartan Books: Washington, 1962; p xvi,616p. 